\title{
An Example of Higher Weight Superpotential Interaction in the Heterotic String on Orbifolds
}

\author{
D.Erdenebayar \\ International Centre for Theoretical Physics, Trieste 34100, Italy \\ and \\ The Institute of Physics and Technology, Ulaanbaatar, Mongolia*
}

November 18, 2018

\begin{abstract}
An explicit orbifold example of the non-zero correlation functions related to the additional contribution to the induced mass term for Higgs particles at low energies is given. We verify that they form finite dimensional representations of the target space modular transformation $S L_{2}(Z)$. This action of the modular group is shown to be consistent with its action on the fixed points set defining the twisted fields.
\end{abstract}

\section{Introduction}

Minimal $\mathrm{N}=1$ supergravity Lagrangian involving two-derivative terms is described by three functions of chiral and anti-chiral superfields $(\Phi, \bar{\Phi})$, which live on a Kähler space. These functions are Kähler potential $K(\Phi, \bar{\Phi})$, chiral superpotential $W(\Phi)$ and the gauge coupling $f(\Phi)$. Kähler potential determines the metric on the Kähler space and therefore the kinetic term of the fields $\Phi$, whereas the superpotential $W$ determines the Yukawa couplings. The fields $\Phi$ in general contain gauge-charged as well as gauge neutral fields. Among

*Permanent Address 
the latter there can be some fields that correspond to flat direction of the potential (i.e. $\frac{\partial V}{\partial \Phi}=0$ identically). Such fields are called moduli fields as they can acquire arbitrary constant vacuum expectation values.

A general feature of the $\mathrm{N}=1$ supergravity models coming from superstring theories is the presence of such moduli fields. They correspond to exactly marginal deformations of the underlying superconformal field theory describing the internal manifold. Besides these moduli fields, which depend on the details of the internal theory, there is a universal field $S$ called dilaton, which is also a flat direction of the potential. In fact the constant v.e.v. of dilaton field determines the string coupling constant. The tree level gauge function $f$ turns out to be only a function of $S$ : the real part of $S$ defines the usual gauge coupling constant and the imaginary part determines the coupling of $F \tilde{F}$ term. Kähler potential $K$ and superpotential $W$ on the other hand are model dependent.

In the case of $(2,2)$ compactification of the Heterotic superstring the gauge group is $E_{6} \times E_{8}$ and the matter fields transform as $\mathbf{2 7}$ or $\overline{\mathbf{2 7}}$ under $E_{6}$ and they are in one-to-one correspondence with the moduli. The Kähler potential has the following power in expansion in the matter fields:

$$
K=G+A^{\alpha} A^{\dot{\alpha}} Z_{\alpha \dot{\alpha}}^{(1,1)}+B^{\nu} B^{\dot{\nu}} Z_{\nu \dot{\nu}}^{(1,2)}+\left(A^{\alpha} B^{\nu} H_{\alpha \nu}+\text { c.c. }\right)+\ldots
$$

where $A$ and $B$ refer to 27 's and $\overline{\mathbf{2 7}}$ 's respectively. The functions $Z_{\alpha \dot{\alpha}}^{(1,1)}$ and $Z_{\nu \dot{\nu}}^{(1,2)}$ determine the kinetic terms and have been studied extensively in the literature [1] and were shown to be related to Yukawa couplings via special geometry relations. $H$ function on the other hand gives rise to mass term (called $\mu$ term) once supersymmetry is broken.

The most convenient way of computing the $H$ function at the tree level is to consider the four-scalar scattering amplitude $\mathcal{A}\left(A^{\alpha}, B^{\nu}, M^{\bar{m}}, M^{\bar{n}}\right)$ involving $\mathbf{2 7}, \overline{\mathbf{2 7}}$ and two antimoduli. In the leading, quadratic order in momenta, there exist in general two contributions to the process. The first, coming from the standard D-term interactions in the effective Lagrangian, gives the Riemann tensor of Kähler geometry, $R_{\alpha \bar{m} \nu \bar{n}}=\nabla_{\bar{m}} \partial_{\bar{n}} H_{\alpha \nu}$. The second contribution exists only in the presence of Yukawa coupling involving $A, B$ and a gauge singlet $s$, and it is not described by the standard supergravity Lagrangian. It involves a two-derivative coupling of two anti-moduli to the auxiliary component of $\bar{s}$. The auxiliary field propagates between this coupling and the Yukawa coupling to $A$ and $B$, producing an effective interaction which contributes to $\mathcal{A}\left(A^{\alpha}, B^{\nu}, M^{\bar{m}}, M^{\bar{n}}\right)$. Such higher-derivative couplings of auxiliary field and scalars are coming from a higher weight F-term in superconformal supergravity. 


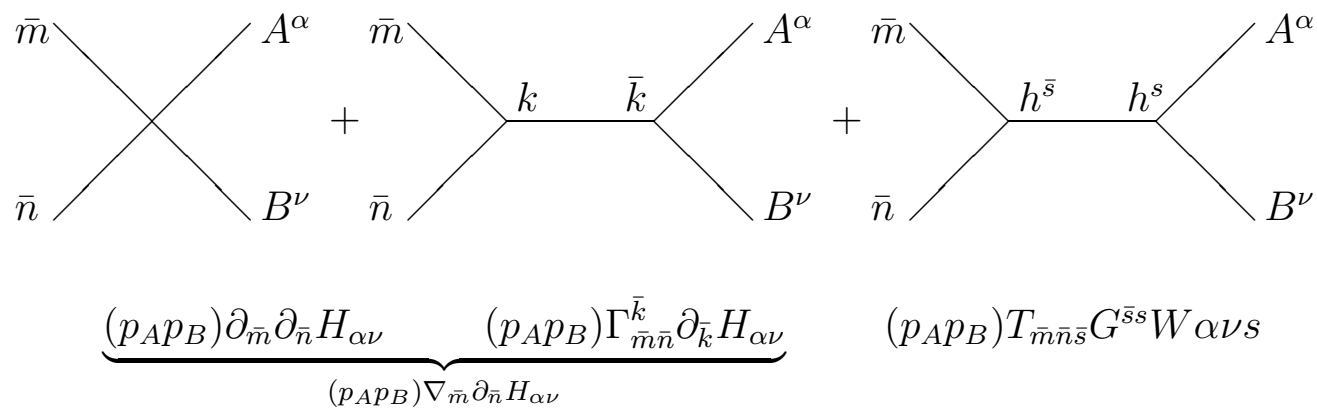

where $h^{s}$ is the auxiliary component of gauge singlet $s$.

Such higher derivative term can be expressed as

$$
\int d^{2} \theta\left(\bar{D}^{2} f_{1}\right)\left(\bar{D}^{2} f_{2}\right)
$$

where $\bar{D}^{2}$ is the chiral projector and $f_{1}$ and $f_{2}$ are two arbitrary functions of $\Phi$ and $\bar{\Phi}$. This would give rise to, among other terms, coupling of the type

$$
\partial \bar{\phi}^{\bar{m}} \partial \bar{\phi}^{\bar{n}} h^{\bar{s}} \underbrace{f_{\bar{m} \bar{n}}^{(1} f_{\bar{s}}^{2)}}_{\equiv T_{\bar{m} \bar{n} \bar{s}}}
$$

Such a term also gives a contribution to the $\mu$-term via the following diagram

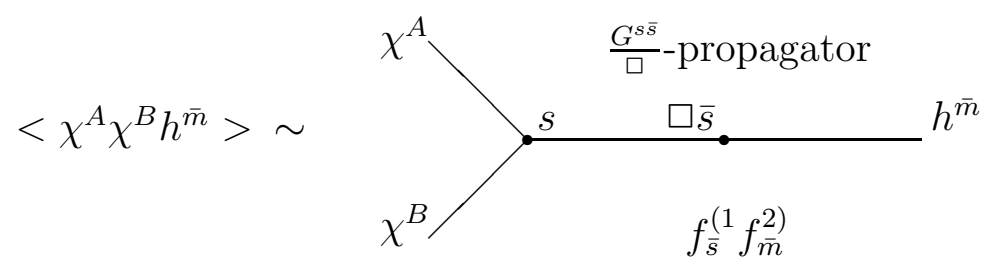

Yukawa coupling $W_{A B s}$

Thus in the event of $h^{\bar{m}}$ acquiring a non-vanishing v.e.v. one gets a mass term of the type

$$
\tilde{\mu}_{A B}=<h^{\bar{m}}>_{\text {v.e.v. }} W_{A B s} G^{s \bar{s}} f_{\bar{s}}^{(1} f_{\bar{m}}^{2)}
$$

which could be of phenomenological interest [3] in the study of low energy physics arising from string theory.

\footnotetext{
${ }^{1}$ for simplicity we will use the language of global supersymmetry although they can be formulated in local supersymmetric way[2]
} 
In [2], by using arguments similar to the ones used in proving special geometry 2], it was shown that for $(2,2)$ models the function $T$ is related to the Yukawa coupling as

$$
\nabla_{[\bar{m}} T_{\bar{n}] \bar{k} \bar{s}}=\nabla_{[\bar{m}}\left(e^{K} \bar{W}_{\bar{n}] \bar{k} \bar{s}}\right)
$$

where we have identified the charged indices $(\alpha, \nu$, etc. $)$ on the right hand side in $W$, with the corresponding moduli indices $n, k$, etc. (Recall that for $(2,2)$ models for every $(1,1)$ moduli there exist a $\mathbf{2 7}$ and for $(1,2)$ a $\overline{\mathbf{2 7}}$.) The right hand side of (1) can be computed by evaluating an amplitude $<\bar{\chi}^{A} \bar{\chi}^{B} M^{\bar{m}} \bar{s}>$.

The purpose of this paper is to compute such amplitudes explicitly in the case of certain orbifold model $]^{\text {] }}$. It turns out that in order to get It turns out that in order to get non-vanishing result all the 4 fields must be twisted fields. Recalling that by $\mathrm{SL}(2, \mathrm{C})$ invariance we can fix 3 points on the sphere, this 4- point correlation function still involves a 2-dimentional world sheet integral over the position of one of the points. We will use the following trick to evaluate this integral. If we take a holomorhic derivative of the r.h.s. of (11) with respect to an untwisted modulus then the result is expected to be a total derivative in the world sheet coordinate:

$$
\begin{gathered}
\nabla_{j} e^{-K} \nabla_{\bar{\imath}}\left(e^{K} W_{\bar{A} \bar{B} \bar{s}}\right)=\nabla_{j}\left(\nabla_{\bar{\imath}}+\partial_{\bar{\imath}} K\right) W_{\bar{A} \bar{B} \bar{s}} \\
=\left[\nabla_{j}, \nabla_{\bar{\imath}}\right] W_{\bar{A} \bar{B} \bar{s}}+G_{j \bar{\imath}} W_{\bar{A} \bar{B} \bar{s}}+\left(\nabla_{\bar{\imath}}+\partial_{\bar{\imath}} K\right) \nabla_{j} W_{\bar{A} \bar{B} \bar{s}}
\end{gathered}
$$

Since for our case $j$ and $\bar{\imath}$ are untwisted and twisted indexes respectively, $G_{j \bar{\imath}}=0$. It is also known that the holomorphic derivative of the Yukawa coupling $\nabla_{j} W_{\bar{A} \bar{B} \bar{s}}$ is zero, so we have

$$
\begin{aligned}
& \nabla_{j} e^{-K} \nabla_{\bar{\imath}}\left(e^{K} W_{\bar{A} \bar{B} \bar{s}}\right)=R_{j \bar{\imath} \bar{A} A^{\prime}} G^{A^{\prime} \bar{A}^{\prime}} W_{\overline{A^{\prime}} \bar{B} \bar{s}} \\
& +R_{j \bar{\imath} \bar{B} B^{\prime}} G^{B^{\prime} \bar{B}^{\prime}} W_{\bar{A} \bar{B}^{\prime} \bar{s}}+R_{j \bar{\imath} \bar{s} s^{\prime}} G^{s^{\prime} \bar{s}^{\prime}} W_{\bar{A} \bar{B} \bar{s}^{\prime}}
\end{aligned}
$$

where the remaining terms correspond to factorized diagrams which show us that the only contribution should come from the boundary of the world sheet

\footnotetext{
${ }^{2}$ i.e. using the $\mathrm{N}=2$ superconformal algebra of the bosonic sector 国

${ }^{3}$ in order to simplify notation we evaluate the amplitudes of holomorphic states instead antiholomorphic ones
} 


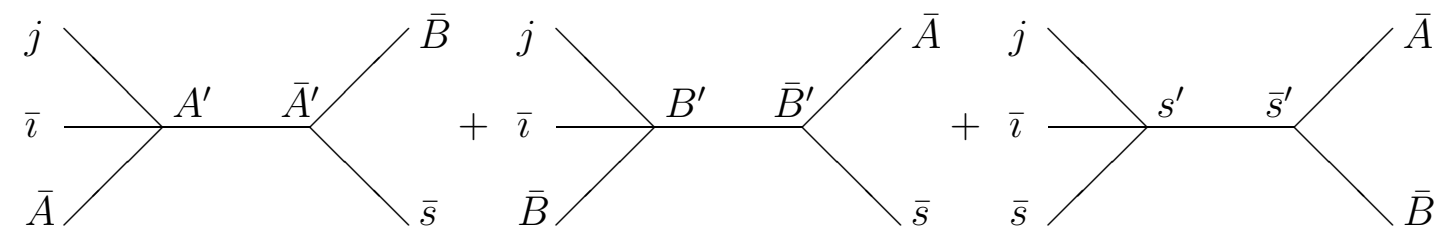

moduli space.

Indeed in the following we will see an explicit example that holomorphic derivative gives rise to a total derivative on the world sheet as indicated by this general argument and this enables us to do the integral explicitely.

The paper is organized as follows. In sect. 2 we derive the 4-point correlation function of the bosonic twist fields in the presence $g, g^{-1}, h$ and $h^{-1}$-twisted sectors using a technique developed in [5]. In sect.3 we give an example of the non-zero correlation functions of the required type $<\overline{27}, 1_{s}, 27,1_{m}>$ and compute them as functions of untwisted moduli (radii, etc.). In sect. 4 we show that they form finite dimensional representation of the duality group $S L_{2}(Z)$, acting on the untwisted moduli space. The action of the duality group is shown to be consistent with the action on the twisted fields characterized by the fixed points set under the orbifold group. In sect.5 using the above mentioned trick we find a differential equation for the correlation function and discuss the uniqueness of its solution. Conclusions are presented in sect.6. Calculation of some useful integrals are given in the appendix.

\section{Correlation function of the bosonic twist fields}

We now present a calculation of a correlation function in which the four twisted states at the points $z_{1}=0, z_{2}=x, z_{3}=1$ and $z_{4}=\infty$ respectively are from $g, g^{-1}, h$ and $h^{-1}$ - twisted sectors. The correlation function to be calculated is

$$
Z_{\text {bos }}\left(z_{i}, \bar{z}_{i}\right) \equiv<\sigma_{g, f_{1}}\left(z_{1}, \bar{z}_{1}\right) \sigma_{g^{-1}, f_{2}}\left(z_{2}, \bar{z}_{2}\right) \sigma_{h, f_{3}}\left(z_{3}, \bar{z}_{3}\right) \sigma_{h^{-1}, f_{4}}\left(z_{4}, \bar{z}_{4}\right)>
$$

The given twist field $\sigma_{g, f}$ determines how $X(z, \bar{z})$ is rotated and translated when it is carried around that operator in the complex $z$-plane:

$$
X\left(e^{2 \pi i} z, e^{-2 \pi i} \bar{z}\right)=\theta^{j} X(z, \bar{z})+v
$$




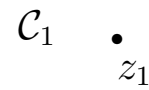

$z_{1}$

$z_{4}$
$\mathcal{C}_{2}$

$z_{3}$

Figure 1: Two independent loops

where $v$ belongs to a coset of $\Lambda$ f which depends on the index $f$. If we split the field $X$ into a classical piece $X_{c l}$ and a quantum fluctuation $X_{q u}$ then the correlation function divides into a sum over classical solutions, times the quantum effective action, both evaluated in the presence of twist fields:

$$
Z_{b o s}=\sum_{<X_{c l}>} e^{-S_{c l}} Z_{q u}
$$

All global information needed to determine both the quantum Green functions and the proper set of classical solutions can be determined from the monodromy conditions for transporting $X_{q u}$ and $X_{c l}$ around collection of fields which have net twist zero.

For our choice of closed loops shown in Fig.1, we have

$$
\oint_{\mathcal{C}_{a}} d X_{q u}=0, \oint_{\mathcal{C}_{a}} d X_{c l}=v_{a}, a=1,2
$$

where

$$
\begin{gathered}
v_{1} \in(1-g)\left(f_{1}-f_{2}+\Lambda_{1}-\Lambda_{2}\right) \\
v_{2} \in(1-g)(1-h)\left(f_{3}-f_{2}+\Lambda_{3}-\Lambda_{2}\right)
\end{gathered}
$$

Taking the closed loop $\mathcal{C}$ to encircle all the vertex operators gives the following constraints for the allowed fixed points:

$$
(1-g)\left(f_{1}-f_{2}+\Lambda_{1}-\Lambda_{2}\right)+(1-h)\left(f_{3}-f_{4}+\Lambda_{3}-\Lambda_{4}\right)=0
$$

\footnotetext{
${ }^{4}$ by $\Lambda$ we denoted hexagonal lattice
} 


\subsection{Quantum part}

The first step in constructing the quantum piece of the correlation function (3) is to find the Green functions in the presence of the four twists

$$
\begin{aligned}
g\left(z, w ; z_{i}\right) & \equiv \frac{<-\frac{1}{2} \partial X(w) \partial \bar{X}(z) \sigma_{g}\left(z_{1}\right) \sigma_{g^{-1}}\left(z_{2}\right) \sigma_{h}\left(z_{3}\right) \sigma_{h^{-1}}\left(z_{4}\right)>}{<\sigma_{g}\left(z_{1}\right) \sigma_{g^{-1}}\left(z_{2}\right) \sigma_{h}\left(z_{3}\right) \sigma_{h^{-1}}\left(z_{4}\right)>} \\
h\left(z, w ; z_{i}\right) \equiv & \frac{<-\frac{1}{2} \partial X(w) \bar{\partial} \bar{X}(\bar{z}) \sigma_{g}\left(z_{1}\right) \sigma_{g^{-1}}\left(z_{2}\right) \sigma_{h}\left(z_{3}\right) \sigma_{h^{-1}}\left(z_{4}\right)>}{<\sigma_{g}\left(z_{1}\right) \sigma_{g^{-1}}\left(z_{2}\right) \sigma_{h}\left(z_{3}\right) \sigma_{h^{-1}}\left(z_{4}\right)>}
\end{aligned}
$$

Using asymptotic conditions as $z \rightarrow w$ and $z, w \rightarrow z_{i}$ we find (we have used the following notation: $g=e^{2 \pi \alpha i}, h=e^{2 \pi \beta i}, a=\alpha-1, b=\beta-1, a+c=-1$ and $b+d=-1)$

$$
\begin{gathered}
g\left(z, w ; z_{i}\right)=\omega_{1}(w) \omega_{2}(z)\left\{(b+1) \frac{\left(w-z_{2}\right)\left(w-z_{4}\right)\left(z-z_{1}\right)\left(z-z_{3}\right)}{(z-w)^{2}}\right. \\
-a \frac{\left(w-z_{1}\right)\left(w-z_{3}\right)\left(z-z_{2}\right)\left(z-z_{4}\right)}{(z-w)^{2}} \\
\left.+(a-b) \frac{\left(w-z_{2}\right)\left(w-z_{3}\right)\left(z-z_{1}\right)\left(z-z_{4}\right)}{(z-w)^{2}}+A\left(z_{i}, \bar{z}_{i}\right)\right\} \\
h\left(z, w ; z_{i}\right)=\omega_{1}(w) \bar{\omega}_{1}(\bar{z}) B\left(z_{i}, \bar{z}_{i}\right)
\end{gathered}
$$

where

$$
\begin{aligned}
& \omega_{1}(z)=\left(z-z_{1}\right)^{a}\left(z-z_{2}\right)^{c}\left(z-z_{3}\right)^{b}\left(z-z_{4}\right)^{d} \\
& \omega_{2}(z)=\left(z-z_{1}\right)^{c}\left(z-z_{2}\right)^{a}\left(z-z_{3}\right)^{d}\left(z-z_{4}\right)^{b}
\end{aligned}
$$

and $A\left(z_{i}, \bar{z}_{i}\right)$ and $B\left(z_{i}, \bar{z}_{i}\right)$ will be determined later using the global monodromy condition (6).

The operator product

$$
-\frac{1}{2} \partial_{z} X \partial_{w} \bar{X} \sim \frac{1}{(z-w)^{2}}+T(z)+\ldots
$$

under limit $w \rightarrow z$ gives us

$$
\begin{gathered}
\frac{\langle T(z) \sigma \ldots \sigma>}{\langle\sigma \ldots \sigma>}=\lim _{w \rightarrow z}\left[g(z, w)-\frac{1}{(z-w)^{2}}\right] \\
=\frac{1}{2} \alpha(1-\alpha)\left(\frac{1}{\left(z-z_{1}\right)}-\frac{1}{\left(z-z_{2}\right)}\right)^{2}+\frac{1}{2} \beta(1-\beta)\left(\frac{1}{\left(z-z_{3}\right)}-\frac{1}{\left(z-z_{4}\right)}\right)^{2}
\end{gathered}
$$




$$
\begin{aligned}
+\beta(1-\alpha) & \left(\frac{1}{\left(z-z_{1}\right)}-\frac{1}{\left(z-z_{2}\right)}\right)\left(\frac{1}{\left(z-z_{3}\right)}-\frac{1}{\left(z-z_{4}\right)}\right) \\
& +\frac{A}{\left(z-z_{1}\right)\left(z-z_{2}\right)\left(z-z_{3}\right)\left(z-z_{4}\right)}
\end{aligned}
$$

Then the operator product

$$
T(z) \sigma\left(z_{2}\right) \sim \frac{h_{\sigma} \sigma\left(z_{2}\right)}{\left(z-z_{2}\right)^{2}}+\frac{\partial_{z_{2}} \sigma\left(z_{2}\right)}{\left(z-z_{2}\right)}+\ldots
$$

under limit $z \rightarrow z_{2}$ gives rise to the differential equation

$$
\begin{aligned}
\partial_{z_{2}} \ln Z_{q u}\left(z_{i}, \bar{z}_{i}\right)=- & \alpha(1-\alpha) \frac{1}{z_{2}-z_{1}}-\beta(1-\alpha)\left(\frac{1}{z_{2}-z_{3}}-\frac{1}{z_{2}-z_{4}}\right) \\
& +\frac{A}{\left(z_{2}-z_{1}\right)\left(z_{2}-z_{3}\right)\left(z_{2}-z_{4}\right)}
\end{aligned}
$$

Using now $S L_{2}(C)$ invariance we fix the location of the four vertex operators: $z_{1}=0, z_{2}=x, z_{3}=1, z_{4} \rightarrow \infty$. Then (11) becomes

$$
\partial_{x} \ln Z_{q u}(x, \bar{x})=-\alpha(1-\alpha) \frac{1}{x}-\beta(1-\alpha) \frac{1}{x-1}-\frac{A}{x(1-x)}
$$

where

$$
\begin{gathered}
Z_{q u}(x, \bar{x})=\lim _{z_{\infty} \rightarrow \infty}\left|z_{\infty}\right|^{2 \beta(1-\alpha)}<\sigma(0) \sigma(x) \sigma(1) \sigma\left(z_{\infty}\right)> \\
A(x, \bar{x})=\lim _{z_{\infty} \rightarrow \infty}\left(-z_{\infty}^{-1}\right) A\left(0, x, 1, z_{\infty}\right)
\end{gathered}
$$

The global monodromy condition (6) gives equation to determine $A$ and $B$ :

$$
\oint_{\mathcal{C}_{a}} d z g(z, w)+\oint_{\mathcal{C}_{a}} d \bar{z} h(\bar{z}, w)=0
$$

which became after fixing $z_{i}$ and dividing by $\omega_{1}(w)$ :

$$
\begin{gathered}
A \oint_{\mathcal{C}_{a}} d z \omega_{2}(z)+B \oint_{\mathcal{C}_{a}} d \bar{z} \bar{\omega}_{1}(\bar{z})=\oint_{\mathcal{C}_{a}} d z \omega_{2}(z) \\
\times\left[-(b+1) \frac{(w-x) z(z-1)}{(z-w)^{2}}+a \frac{w(w-x)(z-x)}{(z-w)^{2}}+(b-a) \frac{(w-x)(w-1) z}{(z-w)^{2}}\right]
\end{gathered}
$$

Choosing $w=1$ we can find that

$$
A \oint_{\mathcal{C}_{1}} d z \omega_{2}(z)+B \oint_{\mathcal{C}_{1}} d \bar{z} \bar{\omega}_{1}(\bar{z})=x(1-x) \frac{d}{d x} \oint_{\mathcal{C}_{1}} d z \omega_{2}(z)
$$


and for $w=0$

$$
A \oint_{\mathcal{C}_{2}} d z \omega_{2}(z)+B \oint_{\mathcal{C}_{2}} d \bar{z} \bar{\omega}_{1}(\bar{z})=x(1-x) \frac{d}{d x} \oint_{\mathcal{C}_{2}} d z \omega_{2}(z)
$$

Solving this equations we find

$$
\begin{gathered}
A=x(1-x) \partial_{x} \ln I(x, \bar{x}) \\
B=\frac{x(1-x) \bar{\gamma}\left(\oint_{\mathcal{C}_{1}} \omega_{2}\right)^{2}}{I(x, \bar{x})} \frac{d \tau}{d x}
\end{gathered}
$$

where

$$
I(x, \bar{x})=\oint_{\mathcal{C}_{1}} \omega_{2} \oint_{\mathcal{C}_{2}} \bar{\omega}_{1}-\oint_{\mathcal{C}_{2}} \omega_{2} \oint_{\mathcal{C}_{1}} \bar{\omega}_{1}=\oint_{\mathcal{C}_{1}} \omega_{2} \oint_{\mathcal{C}_{1}} \bar{\omega}_{1} \bar{\gamma}(\bar{\tau}-\tau)
$$

Substituting (13) into (12) and integrating it we get

$$
\begin{gathered}
Z_{q u}(x, \bar{x})=\operatorname{const} \frac{|x|^{-2 \alpha(1-\alpha)}|1-x|^{-2 \beta(1-\alpha)}}{\left|\oint_{\mathcal{C}_{1}} \omega_{2}\right|^{2} \tau_{2}} \\
=\operatorname{const} \frac{|x|^{-2 \alpha(1-\alpha)}|1-x|^{-2 \alpha(1-\beta)}}{|F(1-\beta, \alpha ; 1 ; x)|^{2} \tau_{2}}
\end{gathered}
$$

where we have used the $x \leftrightarrow \bar{x}$ symmetry of $Z_{q u}(x, \bar{x})$ to fix the $\bar{x}$-dependence of the integration constant. From (14) we find

$$
h\left(z, w \mid z_{i}\right)=\frac{i}{2 \tau_{2}} x(1-x) \omega_{1}(w) \bar{\omega}_{1}(\bar{z}) \frac{\oint_{\mathcal{C}_{1}} \omega_{2}}{\oint_{\mathcal{C}_{1}} \bar{\omega}_{1}} \frac{d \tau}{d x}
$$

\subsection{Classical part}

The $z$ and $\bar{z}$ derivatives of the classical solutions can be written as

$$
\begin{aligned}
& \partial X_{c l}(z)=a \omega_{1}(z), \bar{\partial} X_{c l}(\bar{z})=\bar{b} \bar{\omega}_{2}(\bar{z}) \\
& \partial \bar{X}_{c l}(z)=b \omega_{2}(z), \bar{\partial} \bar{X}_{c l}(\bar{z})=\bar{a} \bar{\omega}_{1}(\bar{z})
\end{aligned}
$$

Substituting this into (6) and solving it we find:

$$
a=\frac{v_{1} \oint_{\mathcal{C}_{2}} \bar{\omega}_{2}-v_{2} \oint_{\mathcal{C}_{1}} \bar{\omega}_{2}}{\oint_{\mathcal{C}_{1}} \omega_{1} \oint_{\mathcal{C}_{2}} \bar{\omega}_{2}-\oint_{\mathcal{C}_{2}} \omega_{1} \oint_{\mathcal{C}_{1}} \bar{\omega}_{2}}
$$




$$
\bar{b}=\frac{v_{1} \oint_{\mathcal{C}_{2}} \omega_{1}-v_{2} \oint_{\mathcal{C}_{1}} \omega_{1}}{\oint_{\mathcal{C}_{1}} \bar{\omega}_{2} \oint_{\mathcal{C}_{2}} \omega_{1}-\oint_{\mathcal{C}_{2}} \bar{\omega}_{2} \oint_{\mathcal{C}_{1}} \omega_{1}}
$$

Normalizing $\omega_{1}$ and $\omega_{2}$ and using definition of $\tau$ given in Appendix we can rewrite

$$
\begin{aligned}
& a=\frac{v_{1}(\bar{\tau}-\alpha) \gamma-v_{2}}{\gamma(\bar{\tau}-\tau)} \\
& \bar{b}=\frac{v_{1}(\tau-\alpha) \gamma-v_{2}}{\gamma(\tau-\bar{\tau})}
\end{aligned}
$$

The classical action is given by

$$
\begin{gathered}
S_{c l}=\frac{1}{4 \pi} \int_{C} d^{2} z\left[R^{2}\left(\partial X_{c l} \bar{\partial} \bar{X}_{c l}+\bar{\partial} X_{c l} \partial \bar{X}_{c l}\right)\right. \\
\left.+i b\left(\partial X_{c l} \bar{\partial} \bar{X}_{c l}-\bar{\partial} X_{c l} \partial \bar{X}_{c l}\right)\right] \\
=\frac{-i}{2 \pi \sqrt{3}}\left[T \int d^{2} z \partial X_{c l} \bar{\partial} \bar{X}_{c l}-\bar{T} \int d^{2} z \bar{\partial} X_{c l} \partial \bar{X}_{c l}\right]
\end{gathered}
$$

where

$$
T=\frac{\sqrt{3}}{2}\left(i R^{2}-b\right)
$$

Using results of Appendix after some algebra, the action for the classical solutions becomes

$$
\begin{aligned}
& S_{c l}= \frac{1}{8 \pi|\gamma|^{2}}\left[R^{2}\left(\left|v_{1} \gamma\right|^{2} \tau_{2}+\frac{1}{\tau_{2}}\left|v_{1} \gamma\left(\tau_{1}-\alpha\right)+v_{2}\right|^{2}\right)\right. \\
&\left.-b\left(\left|v_{1} \gamma\right|^{2}(\bar{\alpha}-\alpha)-v_{1} \gamma \bar{v}_{2}+\bar{v}_{1} \bar{\gamma} v_{2}\right)\right]
\end{aligned}
$$

where

$$
\begin{gathered}
\tau(x) \equiv \tau_{1}+i \tau_{2}=\frac{\oint_{\mathcal{C}_{2}} \omega_{1}}{\gamma \oint_{\mathcal{C}_{1}} \omega_{1}}-\alpha \\
=\frac{i}{2 \sqrt{3}}\left(\frac{\sqrt{3}}{\pi}(1-x)^{1 / 6} \frac{\Gamma\left(\frac{1}{2}\right) \Gamma\left(\frac{2}{3}\right)}{\Gamma\left(\frac{7}{6}\right)} \frac{F\left(\frac{2}{3}, \frac{1}{2} ; \frac{7}{6} ; 1-x\right)}{F\left(\frac{1}{2}, \frac{1}{3} ; 1 ; x\right)}+\frac{1}{2}\right)
\end{gathered}
$$

The classical part of the correlation function is given by

$$
Z_{c l}=\sum_{v_{1}, v_{2}} e^{-S_{c l}}
$$


where sum is over cosets $v_{1}$ and $v_{2}$ determined in (17). Substituting $v_{1}=$ $\gamma\left(\epsilon_{1}+\Lambda\right)$ and $v_{2}=\gamma\left(\epsilon_{2}+\Lambda\right)$ we found that $\epsilon_{2}=f_{23}$ f and $\epsilon_{1}$ satisfies the constraint

$$
\epsilon_{1}+\Lambda=\frac{1}{1-h}\left(f_{12}+\Lambda\right) \bigcap\left(\frac{1}{1-g}\left(f_{34}+\Lambda\right)\right.
$$

After Poisson resummation on $v_{2}$ we get

$$
Z_{c l}=\frac{8 \pi|\gamma|^{2}}{R^{2}} \tau_{2} \sum_{v \in \Lambda, p \in \Lambda^{*}} e^{2 \pi i\left(\epsilon_{2}-\gamma \alpha v-\gamma \alpha \epsilon_{1}\right) p} w_{L}^{Q} \bar{w}_{R}^{Q}
$$

where $w=e^{i \pi \tau}, \bar{w}=e^{-i \pi \bar{\tau}}$ and

$$
\begin{aligned}
& Q_{L}=\left|\frac{p}{R}+\frac{\left(v+\epsilon_{1}\right) \gamma}{2 R}\left(R^{2}-i b\right)\right|^{2} \\
& Q_{R}=\left|\frac{p}{R}-\frac{\left(v+\epsilon_{1}\right) \gamma}{2 R}\left(R^{2}+i b\right)\right|^{2}
\end{aligned}
$$

\section{The correlation function $<\overline{27}, 1_{s}, 27,1_{m}>$}

We start from $E_{8} \times E_{8}$ heterotic string compactified on the 6-dimensional orbifold, which is a direct sum of three two- dimensional orbifolds:

$$
Z_{6}=Z_{2}^{(6)} \times Z_{2}^{(3)} \times Z_{2}^{(2)}
$$

where the lattices for the first two are hexagonal (we denote it by $\Lambda$ ) and for the third is arbitrary. Point groups are generated by rotations $\theta$ of order 6,3 and 2 respectively.

To simplify calculations is useful to bosonize all fermions. Using fermionic charge conservation, we found the states $\overline{27}, 27,1_{m}$ and $1_{s}$, whose correlation function of the type $\left\langle f, f, b, b>\right.$ is not zero. The states $\overline{27}$ and $1_{s}$ come from $g^{2}, g^{-2}$ twisted sectors respectively and we choose them to be in the Ramond sector. States 27 and $1_{m}$ come from $g^{3}$ twisted sector and they are in the Neveu-Schwarz sector.

Vertex operators for states are

$$
\begin{aligned}
\overline{27}: V_{-1 / 2}\left(z_{1}\right) & =e^{-\phi / 2} e^{i \alpha H} e^{i p \tilde{H}} \sigma^{1} \sigma^{2} \sigma^{3}\left(z_{1}\right) \mid B> \\
\alpha & =\left(-\frac{1}{6}, \frac{1}{6},-\frac{1}{2}, \frac{1}{2},-\frac{1}{2}\right) \\
p & =\left(\frac{2}{3}, \frac{1}{3}, 0 \mid 1,0^{4}\right)
\end{aligned}
$$

\footnotetext{
${ }^{5}$ we used notation $f_{12}=f_{1}-f_{2}$,etc.

${ }^{6}$ for descripsion of orbifolds and notation see [5]
} 


$$
\begin{gathered}
1_{s}: V_{-1 / 2}\left(z_{2}\right)=e^{-\phi / 2} e^{i \alpha H} e^{i p \tilde{H}} \sigma^{1} \sigma^{2} \sigma^{3}\left(z_{2}\right) \mid B> \\
\alpha=\left(\frac{1}{6},-\frac{1}{6},-\frac{1}{2},-\frac{1}{2}, \frac{1}{2}\right) \\
p=\left(-\frac{2}{3},-\frac{1}{3}, 1 \mid 0^{5}\right) \\
27: V_{-1}\left(z_{3}\right)=e^{-\phi} e^{i \alpha H} e^{i p \tilde{H}} \sigma^{1} \sigma^{2} \sigma^{3}\left(z_{3}\right) \\
(|0>o r| C>)=\left(\frac{1}{2}, 0, \frac{1}{2}, 0,0\right) \\
p=\left(-\frac{1}{2}, 0,-\frac{1}{2} \mid-1,0^{4}\right) \\
1_{m}: V_{0}\left(z_{4}\right)=\lim _{w \rightarrow z_{4}} \oint_{C_{z_{4}}} d \bar{z}\left(e^{\phi} e^{-i H_{1}} \partial X^{1}\right)(w)\left(e^{i \tilde{H}_{1}} \bar{\partial} \bar{X}^{1}\right)(\bar{z}) \\
\quad\left(e^{-\phi} e^{i \alpha H} e^{i p \tilde{H}} \sigma^{1} \sigma^{2} \sigma^{3}\right)\left(z_{4}\right)(\mid 0>\text { or } \mid C>) \\
=\left(\frac{1}{2}, 0, \frac{1}{2}, 0,0\right) \\
=\left(-\frac{1}{2}, 0,-\frac{1}{2} \mid 0^{5}\right)
\end{gathered}
$$

In the expression for the state $1_{m}$ we leave only non-vanishing component of $T_{F}$. The ground states $\mid B>$ and $\mid C>$ are eigenstates of the group action $g$ and they are linear combination of the fixed points:

$$
\begin{gathered}
|B>=| \kappa_{1}>-\mid \kappa_{2}> \\
|C>=| \rho_{1}>+\left|\rho_{2}>+\right| \rho_{3}>
\end{gathered}
$$

where $\kappa_{1}, \kappa_{2}$ are the fixed points for the $2 \pi / 3$ rotation and $\rho_{1}, \rho_{2}, \rho_{3}$ are the fixed points for the $\pi$ rotation on the hexagonal lattice $\Lambda$ (see Fig.2). The correlation functions with different ground states form the multiplet

$$
\left(\begin{array}{c}
<B, B, O, O> \\
<B, B, C, O> \\
<B, B, O, C> \\
<B, B, C, C>
\end{array}\right)
$$

under the modular group transformations, which we will show later. 


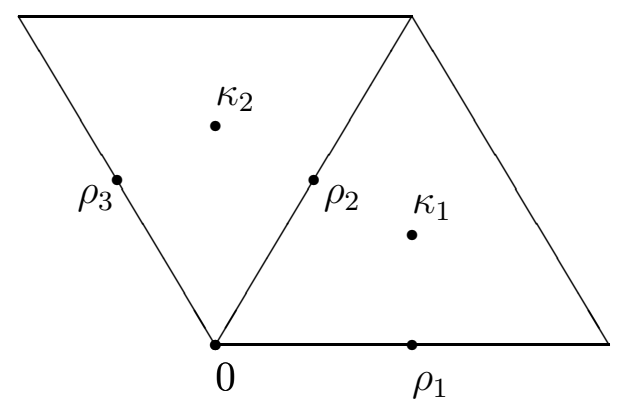

Figure 2: The fixed points for the $\frac{2 \pi}{3}$ and $\frac{2 \pi}{4}$ rotations on the hexagonal lattice $\Lambda$

The correlation function $<\overline{27}, 1_{s}, 27,1_{m}>$ is

$$
\begin{gathered}
Z(x, \bar{x})=<V_{-1 / 2}(0) V_{-1 / 2}(x) V_{-1}(1) V_{0}\left(z_{\infty}\right)> \\
=\lim _{w \rightarrow z_{4}} \oint_{C_{z_{4}}} d \bar{z}<e^{-\phi / 2}(0) e^{-\phi / 2}(x) e^{-\phi}(1) e^{-\phi}\left(z_{\infty}\right) e^{\phi}(w)> \\
\times<e^{i \sigma}(0) e^{i \sigma}(1) e^{i \sigma}\left(z_{\infty}\right) \times c . c .> \\
\times<e^{i \alpha H}(0) e^{i \alpha H}(x) e^{i \alpha H}(1) e^{i \alpha H}\left(z_{\infty}\right) e^{-i H_{1}}(w)> \\
\times<e^{i p \tilde{H}}(0) e^{i p \tilde{H}}(\bar{x}) e^{i p \tilde{H}}(1) e^{i p \tilde{H}}\left(z_{\infty}\right) e^{i \tilde{H}_{1}}(\bar{z})> \\
\times<\sigma_{1 / 3}^{1}(0) \sigma_{-1 / 3}^{1}(x, \bar{x}) \sigma_{1 / 2}^{1}(1) \sigma_{1 / 2}^{1}\left(z_{\infty}\right) \partial X^{1}(w) \bar{\partial} \bar{X}^{1}(\bar{z})> \\
\times<\sigma_{2 / 3}^{2}(0) \sigma_{-2 / 3}^{2}(x, \bar{x})><\sigma_{1 / 2}^{3}(1) \sigma_{1 / 2}^{3}\left(z_{\infty}\right)>
\end{gathered}
$$

Here we had set space-time momenta to zero.

Correlations of all fields except the bosonic twists are found from the formula:

$$
<e^{i k_{1} X}\left(z_{1}\right) \ldots e^{i k_{n} X}\left(z_{n}\right)=\prod_{1 \leq i<j \leq n}\left(z_{i}-z_{j}\right)^{k_{i} k_{j}}
$$

The correlation of the bosonic twist fields is splitted into a sum of classical and quantum parts.

$$
\begin{gathered}
<\partial X(w) \bar{\partial} \bar{X}(\bar{z}) \sigma \ldots \sigma>=<\partial X_{c l}(w) \bar{\partial} \bar{X}_{c l}(\bar{z}) \sigma \ldots \sigma> \\
+<\partial X_{q u}(w) \bar{\partial} \bar{X}_{q u}(\bar{z}) \sigma \ldots \sigma>
\end{gathered}
$$

In order to determine the classical part we will use the following trick. Let's take derivative of $Z_{b o s}$ with respect to $T$ :

$$
\frac{\partial}{\partial T} Z_{b o s}=-\frac{1}{4 \pi \sqrt{3}} \sum_{<X_{c l}>} \int d^{2} z \partial X_{c l}(z) \wedge \bar{\partial} \bar{X}_{c l}(\bar{z}) Z_{q u} e^{-S_{c l}}
$$




$$
=\frac{i \tau_{2}}{2 \pi \sqrt{3}} \oint_{\mathcal{C}_{1}} \omega_{1} \oint_{\mathcal{C}_{1}} \bar{\omega}_{1} \sum_{<X_{c l}>}|a|^{2} Z_{q u} e^{-S_{c l}}
$$

Then we see that

$$
\begin{gathered}
<\partial X(w)_{c l} \bar{\partial} \bar{X}_{c l}(\bar{z}) \sigma \ldots \sigma>=\omega_{1}(w) \bar{\omega}_{1}(\bar{z}) \sum|a|^{2} Z_{q u} e^{-S_{c l}} \\
=\frac{2 \pi \sqrt{3}}{i \tau_{2}} \frac{\omega_{1}(w) \bar{\omega}_{1}(\bar{z})}{\oint_{\mathcal{C}_{1}} \omega_{1} \oint_{\mathcal{C}_{1}} \bar{\omega}_{1}} \frac{\partial}{\partial T} Z_{b o s}
\end{gathered}
$$

The quantum part $<\partial X_{q u}(w) \bar{\partial} \bar{X}_{q u}(\bar{z}) \sigma \ldots \sigma>$ is found from (17) and (10)

$$
\begin{aligned}
& <\partial X_{q u}(w) \bar{\partial} \bar{X}_{q u}(\bar{z}) \sigma \ldots \sigma>=-2 h(\bar{z}, w) Z_{q u} Z_{c l} \\
& =x(1-x) \frac{\omega_{1}(w) \bar{\omega}_{1}(\bar{z})}{i \tau_{2}, \oint_{\mathcal{C}_{1}} \omega_{1} \oint_{\mathcal{C}_{1}} \bar{\omega}_{1}} \frac{\partial \tau}{\partial x} \oint_{\mathcal{C}_{1}} \omega_{2} \oint_{\mathcal{C}_{1}} \omega_{1} Z_{b o s}
\end{aligned}
$$

Substituting (25) and (26) in (23) we have

$$
\begin{gathered}
<\partial X(w) \bar{\partial} \bar{X}(\bar{z}) \sigma \ldots \sigma>=\frac{\omega_{1}(w) \bar{\omega}_{1}(\bar{z})}{\tau_{2}\left|\oint_{\mathcal{C}_{1}} \omega_{1}\right|^{2}} \frac{1}{R^{2}} \frac{\partial}{\partial T}\left(R^{2} Z_{b o s}\right) \\
=\operatorname{const} \frac{\omega_{1}(w) \bar{\omega}_{1}(\bar{z})}{\tau_{2}|F(1 / 2,1 / 3,1, x)|^{2}} \\
\times \frac{1}{R^{2}} \frac{\partial}{\partial T}\left[\frac{|x|^{-4 / 9}|1-x|^{-1 / 3}}{|F(1 / 2,1 / 3,1, x)|^{2}} \sum_{v \in \Lambda, p \in \Lambda^{*}} e^{2 \pi i\left(\epsilon_{2}-\gamma \alpha v-\gamma \alpha \epsilon_{1}\right) p} w^{Q_{L}} \bar{w}^{Q_{R}}\right]
\end{gathered}
$$

The full correlation function (22) is then integrated over the complex plane and gives the scattering apmlitude for four massless twisted states:

$$
\begin{aligned}
A_{\epsilon_{j}}(T, \bar{T})=\int d^{2} x Z(x, \bar{x})=\mathrm{const} \int d^{2} x \frac{1}{\tau_{2}} \frac{x^{-1}(1-x)^{-5 / 6} \bar{x}^{-1}(1-\bar{x})^{-1 / 3}}{|F(1 / 2,1 / 3 ; 1 ; x)|^{4}} \\
\times \frac{1}{R^{2}} \frac{\partial}{\partial T} \sum_{v \in \Lambda, p \in \Lambda^{*}} e^{2 \pi i\left(\epsilon_{2}-\gamma \alpha v-\gamma \alpha \epsilon_{1}\right) p} w^{Q_{L}} \bar{w}^{Q_{R}}
\end{aligned}
$$

Recalling that

$$
\frac{d \tau}{d x}=\frac{1}{2 \pi i} \frac{x^{-1}(1-x)^{-5 / 6}}{F(1 / 2,1 / 3 ; 1 ; x)^{2}}
$$

we rewrite

$$
A_{\epsilon_{j}}(T, \bar{T})=\frac{1}{R^{2}} \int d^{2} x \frac{1}{\tau_{2}} \frac{d \tau}{d x} \frac{d \bar{\tau}}{d \bar{x}}(1-\bar{x})^{1 / 2} \frac{\partial}{\partial T} C\left(\epsilon_{1}, \epsilon_{2}\right)
$$

where

$$
C\left(\epsilon_{1}, \epsilon_{2}\right)=\sum_{v \in \Lambda, p \in \Lambda^{*}} e^{2 \pi i\left(\epsilon_{2}-\gamma \alpha v-\gamma \alpha \epsilon_{1}\right) p} w^{Q_{L}} \bar{w}^{Q_{R}}
$$




\section{Modular transformation}

If we decompose $v=v_{0}+\rho$ and $p=p_{0}+c+\mu$, where $v_{0} \in 2 \Lambda, \rho \in \Lambda / 2 \Lambda$, $p_{0} \in 2 \gamma \Lambda^{*}, \mu \in \gamma \Lambda^{*} / 2 \gamma \Lambda^{*}, c \in \Lambda^{*} / \gamma \Lambda^{*}$ then (29) becomes

$$
\begin{gathered}
C\left(\epsilon_{1}, \epsilon_{2}\right)=\sum_{\rho, c, \mu} \sum_{v_{0}, p_{0}} e^{2 \pi i\left(\epsilon_{2}-\gamma \alpha\left(v_{0}+\rho\right)-\gamma \alpha \epsilon_{1}\right)\left(p_{0}+c+\mu\right)} w^{Q_{L}} \bar{w}^{Q_{R}} \\
=\sum_{\rho, c, \mu} e^{2 \pi i\left(\epsilon_{2}-\alpha \gamma \epsilon_{1}\right) c} e^{\left.-2 \pi i \alpha \gamma\left(\rho c+\epsilon_{1}\right) \mu\right)} Z\left(\epsilon_{1}+\rho, c+\mu\right)
\end{gathered}
$$

where

$$
Z\left(\epsilon_{1}+\rho, c+\mu\right)=\sum_{v_{0}, p_{0}} w^{Q_{L}} \bar{w}^{Q_{R}}
$$

FFrom (30) we find it's Fourier transformation

$$
\sum_{\epsilon_{2}} C\left(\epsilon_{1}, \epsilon_{2}\right) e^{-2 \pi i\left(\epsilon_{2}-\alpha \gamma \epsilon_{1}\right) \tilde{c}}=12 \sum_{\rho, \tilde{\mu}} e^{\left.-2 \pi i \alpha \gamma\left(\rho \tilde{c}+\epsilon_{1}\right) \tilde{\mu}\right)} Z\left(\epsilon_{1}+\rho, \tilde{c}+\tilde{\mu}\right)
$$

The correlation function should be invariant under modular transformations

$$
T \rightarrow \frac{a T+b}{c T+d}
$$

where $T=\frac{\sqrt{3}}{2}\left(i R^{2}-b\right)$ and $a, b, c, d$ form an element of $S L_{2}(Z)$.

Modular transformations can be generated by two basic transformations:

$$
\mathcal{S}: T \rightarrow-\frac{1}{T} \Leftrightarrow\left\{\begin{array}{l}
R^{2}+i b \rightarrow \frac{4}{3\left(R^{2}+i b\right)} \\
R^{2}-i b \rightarrow \frac{4}{3\left(R^{2}-i b\right)}
\end{array} \Rightarrow R \rightarrow \frac{2 R}{\sqrt{3}\left(R^{4}+b^{2}\right)^{1 / 2}}\right.
$$

and

$$
\mathcal{T}: T \rightarrow T+1 \Leftrightarrow\left\{\begin{array}{l}
b \rightarrow b-\frac{2}{\sqrt{3}} \\
R \rightarrow R
\end{array}\right.
$$

\subsection{S-transformation}

We find that under S-transformation

$$
Z\left(\epsilon_{1}+\rho, c+\mu\right) \stackrel{\mathcal{S}}{\rightarrow} Z\left(\tilde{\epsilon}_{1}+\tilde{\rho}, \tilde{c}+\tilde{\mu}\right)
$$

where

$$
\begin{gathered}
\tilde{v}_{0}=\frac{\theta}{2} p_{0}, \tilde{\epsilon}_{1}=\frac{\theta}{2} c, \tilde{\rho}=\frac{\theta}{2} \mu \\
\tilde{p}_{0}=2 v_{0}, \tilde{c}=2 \epsilon_{1}, \tilde{\mu}=2 \rho
\end{gathered}
$$


After some calculations we find

$$
C\left(\epsilon_{1}, \epsilon_{2}\right) \stackrel{\mathcal{S}}{\rightarrow} \frac{1}{12} \sum_{\tilde{\epsilon}_{1}, \tilde{\epsilon}_{2}} e^{4 \pi i\left(\theta^{-1} \tilde{\epsilon}_{1} \epsilon_{2}-\epsilon_{1} \tilde{\epsilon}_{2}-\epsilon_{1} \theta \tilde{\epsilon}_{1}\right)} C\left(\tilde{\epsilon}_{1}, \tilde{\epsilon}_{2}\right)
$$

Knowing that the solutions of (20) for the case $g=\theta^{2}, h=\theta^{3}\left(\theta=e^{2 \pi i / 6}\right)$ is

$$
\epsilon_{2}=f_{23}, \epsilon_{1}=\theta^{2} f_{34}-f_{12}
$$

and substituting them back we get

$$
<f_{1}, f_{2}, f_{3}, f_{4}>\stackrel{\mathcal{S}}{\rightarrow} \frac{1}{12} \sum e^{4 \pi i\left(f_{1} \tilde{f}_{1}-f_{2} \tilde{f}_{2}-f_{3} \theta \tilde{f}_{3}-f_{4} \theta \tilde{f}_{4}\right)}<\tilde{f}_{1}, \tilde{f}_{2}, \tilde{f}_{3}, \tilde{f}_{4}>
$$

from which we can deduce that the modular transformation acts on states separately:

$$
\begin{aligned}
& <f_{1}>\stackrel{\mathcal{S}}{\rightarrow} \frac{1}{\sqrt{3}} e^{4 \pi i f_{1} \tilde{f}_{1}}<\tilde{f}_{1}> \\
& <f_{2}>\stackrel{\mathcal{S}}{\rightarrow} \frac{1}{\sqrt{3}} e^{-4 \pi i f_{2} \tilde{f}_{2}}<\tilde{f}_{2}> \\
& <f_{3}>\stackrel{\mathcal{S}}{\rightarrow} \frac{1}{2} e^{-4 \pi i f_{3} \theta \tilde{f}_{3}}<\tilde{f}_{3}> \\
& <f_{4}>\stackrel{\mathcal{S}}{\rightarrow} \frac{1}{2} e^{-4 \pi i f_{4} \theta \tilde{f}_{4}}<\tilde{f}_{4}>
\end{aligned}
$$

For the physical states the transformation become

$<B>\stackrel{\mathcal{S}}{\rightarrow}-i<B>$-for $g^{2}$ twisted sector

$<B>\stackrel{\mathcal{S}}{\rightarrow} i<B>$-for $g^{-2}$ twisted sector and

$$
\left(\begin{array}{c}
<O> \\
\frac{1}{\sqrt{3}}\langle C>
\end{array}\right) \stackrel{\mathcal{S}}{\rightarrow} \frac{1}{2}\left(\begin{array}{cc}
1 & \sqrt{3} \\
\sqrt{3} & -1
\end{array}\right)\left(\begin{array}{c}
<O> \\
\frac{1}{\sqrt{3}}<C>
\end{array}\right) \text {-for } g^{3} \text { twisted sector }
$$

Finally we get that the multiplet of the correlation functions transforms

$$
\left(\begin{array}{c}
<B, B, O, O> \\
<B, B, C, O> \\
<B, B, O, C> \\
<B, B, C, C>
\end{array}\right) \stackrel{\mathcal{S}}{\rightarrow} \frac{1}{4}\left(\begin{array}{cccc}
1 & 1 & 1 & 1 \\
3 & -1 & 3 & -1 \\
3 & 3 & -1 & -1 \\
9 & -3 & -3 & 1
\end{array}\right)\left(\begin{array}{c}
<B, B, O, O> \\
<B, B, C, O> \\
<B, B, O, C> \\
<B, B, C, C>
\end{array}\right)
$$

Defining the quantity $G(T, \bar{T})=R^{2} A_{\epsilon_{j}}(T, \bar{T})$ we find the following transformation

$$
G\left(\begin{array}{l}
<O, O> \\
<C, O> \\
<O, C> \\
<C, C>
\end{array}\right) \stackrel{\mathcal{S}}{\rightarrow} \frac{T^{2}}{4}\left(\begin{array}{cccc}
1 & 1 & 1 & 1 \\
3 & -1 & 3 & -1 \\
3 & 3 & -1 & -1 \\
9 & -3 & -3 & 1
\end{array}\right) G\left(\begin{array}{c}
<O, O> \\
<C, O> \\
<O, C> \\
<C, C>
\end{array}\right)
$$




\subsection{T-transformation}

Under T-transformation

$$
Z\left(\epsilon_{1}+\rho, c+\mu\right) \stackrel{\mathcal{T}}{\rightarrow} Z\left(\epsilon_{1}+\rho, \tilde{c}+\tilde{\mu}\right)
$$

where

$$
\tilde{p}_{0}=p_{0}+2 \theta v_{0}, \tilde{c}=c+2 \theta \epsilon_{1}, \tilde{\mu}=\mu+2 \theta \rho
$$

After some calculations we find

$$
C\left(\epsilon_{1}, \epsilon_{2}\right) \stackrel{\mathcal{T}}{\rightarrow} e^{-2 \pi i\left(2 \epsilon_{2}+\theta \epsilon_{1}\right) \theta \epsilon_{1}} C\left(\epsilon_{1}, \epsilon_{2}\right)
$$

and

$$
<f_{1}, f_{2}, f_{3}, f_{4}>\stackrel{\mathcal{T}}{\rightarrow} e^{2 \pi i\left(-f_{1} f_{1}+f_{2} f_{2}+f_{3} f_{3}+f_{4} f_{4}\right)}<f_{1}, f_{2}, f_{3}, f_{4}>
$$

From (33) we can deduce, as in the previous case, that the modular transformation acts on states separately:

$$
\begin{aligned}
& <f_{1}>\stackrel{\mathcal{T}}{\rightarrow} e^{-2 \pi i f_{1} f_{1}}<f_{1}> \\
& <f_{2}>\stackrel{\mathcal{T}}{\rightarrow} e^{2 \pi i f_{2} f_{2}}<f_{2}> \\
& <f_{3}>\stackrel{\mathcal{T}}{\rightarrow} e^{2 \pi i f_{3} f_{3}}<f_{3}> \\
& <f_{4}>\stackrel{\mathcal{T}}{\rightarrow} e^{2 \pi i f_{4} f_{4}}<f_{4}>
\end{aligned}
$$

which for physical states become

$<B>\stackrel{\mathcal{T}}{\rightarrow} e^{2 \pi i / 3}<B>$-for $g^{2}$ twisted sector

$<B>\stackrel{\mathcal{T}}{\rightarrow} e^{-2 \pi i / 3}<B>$-for $g^{-2}$ twisted sector

and

$$
\left(\begin{array}{c}
<O> \\
<C>
\end{array}\right) \stackrel{\mathcal{T}}{\rightarrow}\left(\begin{array}{cc}
1 & 0 \\
0 & -1
\end{array}\right)\left(\begin{array}{l}
<O> \\
<C>
\end{array}\right) \text {-for } g^{3} \text { twisted sector }
$$

The multiplet of the correlation functions transforms as follows

$$
\left(\begin{array}{c}
<B, B, O, O> \\
<B, B, C, O> \\
<B, B, O, C> \\
<B, B, C, C>
\end{array}\right) \rightarrow \mathcal{T}_{\rightarrow}\left(\begin{array}{cccc}
1 & 0 & 0 & 0 \\
0 & -1 & 0 & 0 \\
0 & 0 & -1 & 0 \\
0 & 0 & 0 & 1
\end{array}\right)\left(\begin{array}{c}
<B, B, O, O> \\
<B, B, C, O> \\
<B, B, O, C> \\
<B, B, C, C>
\end{array}\right)
$$

Under $\mathcal{T}$-transformation $G(T, \bar{T})$ transforms as

$$
G\left(\begin{array}{c}
<O, O> \\
<C, O> \\
<O, C> \\
<C, C>
\end{array}\right) \stackrel{\mathcal{T}}{\rightarrow}\left(\begin{array}{cccc}
1 & 0 & 0 & 0 \\
0 & -1 & 0 & 0 \\
0 & 0 & -1 & 0 \\
0 & 0 & 0 & 1
\end{array}\right) G\left(\begin{array}{c}
<O, O> \\
<C, O> \\
<O, C> \\
<C, C>
\end{array}\right)
$$


Since $\mathcal{S}$ and $\mathcal{T}$ transformations generate all modular group, from (32) and (34) we deduce that the correlation functions (28) form finite dimensional representation of the duality group $S L_{2}(Z)$.

\section{Integration of the correlation function}

In order to integrate the amplitude (28) we take a derivative of $G(T, \bar{T})$ with respect to $\bar{T}$ and refering to the following formula[9]

$$
\frac{\partial^{2}}{\partial \bar{T} \partial T}\left(w^{Q_{L}} \bar{w}^{Q_{R}}\right)=-\frac{4 \tau_{2}}{(T-\bar{T})^{2}} \partial_{\tau} \partial_{\bar{\tau}}\left(w^{Q_{L}} \bar{w}^{Q_{R}} \tau_{2}\right)
$$

we find

$$
\begin{gathered}
\frac{\partial}{\partial \bar{T}} G(T, \bar{T})=\int d^{2} x \frac{1}{\tau_{2}} \frac{d \tau}{d x} \frac{d \bar{\tau}}{d \bar{x}}(1-\bar{x})^{1 / 2} \frac{\partial^{2}}{\partial \bar{T} \partial T} C(x, \bar{x}) \\
=-\frac{4}{(T-\bar{T})^{2}} \int d^{2} x \partial_{x}\left[\frac{d \bar{\tau}}{d \bar{x}}(1-\bar{x})^{1 / 2} \partial_{\bar{\tau}}\left(C(x, \bar{x}) \tau_{2}\right)\right]
\end{gathered}
$$

The later integral can be written as a surface integral, turning out to be a sum of the surface integrals at $0,1, \infty$.

$$
\left.\frac{\partial}{\partial \bar{T}} G(T, \bar{T})=-\frac{2 \sqrt{2} \pi}{(T-\bar{T})^{2}} \sum_{x_{0} \in\{0,1, \infty\}}\left(\bar{x}-\bar{x}_{0}\right)^{1 / 2} \frac{d \bar{\tau}}{d \bar{x}}(1-\bar{x})^{1 / 2} \partial_{\bar{\tau}}\left(C(x, \bar{x}) \tau_{2}\right)\right]_{x=x_{0}, \bar{x}=\bar{x}_{0}}
$$

The first term of the above sum at $x_{0}=1$ does not give any contribution while the terms at $x_{0}=0$ and $x_{0}=\infty$ give rise to a non-zero result. Recalling $\tau \rightarrow \frac{i}{2 \pi} \log \left(\frac{12}{x}\right)$ then $x \rightarrow 0$ we find that the second term of the sum at $x_{0}=0$ equal

$$
-\frac{2 \sqrt{2} \pi i}{(T-\bar{T})^{2}}
$$

where the only non-zero contribution is from sector $Q_{L}=Q_{R}=0$.

To find the contribution at $x_{0}=\infty$ the integral over $x$-plane is written as an integral over $\tau$-plane:

$$
\begin{aligned}
& \left.-\frac{4}{(T-\bar{T})^{2}} \int d^{2} \tau \frac{1}{\tau_{2}}(1-\bar{x}(\bar{\tau}))^{1 / 2} \partial_{\tau} \partial_{\bar{\tau}}\left(C(\tau, \bar{\tau}) \tau_{2}\right)\right] \\
= & \left.-\frac{2 \sqrt{2} \pi}{(T-\bar{T})^{2}}\left(\bar{\tau}-\bar{\tau}_{0}\right)(1-\bar{x}(\bar{\tau}))^{1 / 2} \partial_{\bar{\tau}}\left(C(\tau, \bar{\tau}) \tau_{2}\right)\right]_{\tau=\tau_{0}}
\end{aligned}
$$


where

$$
\tau_{0}=-\frac{1}{2}+\frac{i}{4 \sqrt{3}}
$$

Expanding the functions $(1-\bar{x}(\bar{\tau}))^{1 / 2}$ and $\partial_{\bar{\tau}}\left(C(\tau, \bar{\tau}) \tau_{2}\right)$ in powers of $\left(\bar{\tau}-\bar{\tau}_{0}\right)$ we find that the third term of the sum at $x_{0}=\infty$ is

$$
-\frac{\pi}{(T-\bar{T})^{2}} \frac{b^{3} \sqrt{2}}{12 \sqrt{3}} \sum_{v \in \Lambda, p \in \Lambda^{*}} e^{2 \pi i\left(\epsilon_{2}-\gamma \alpha v-\gamma \alpha \epsilon_{1}\right) p} w^{Q_{L}} \bar{w}^{Q_{R}} P\left(Q_{R}\right)
$$

where

$$
P\left(Q_{R}\right)=\frac{\pi^{3}}{8 \sqrt{3}} Q_{R}^{3}-\frac{9 \pi^{2}}{4} Q_{R}^{2}+9 \sqrt{3} \pi Q_{R}-18
$$

The first term vanishes according to the general argument mentioned in above. The corresponding term is $R_{\bar{T} m \overline{27} \overline{27}^{\prime *} G_{\overline{27}}{ }^{\prime *} \overline{27}^{\prime}} W_{\overline{27}^{\prime}}{ }_{27 s}$ and it happens in our model that there is not a such Yukawa coupling. The reason why the second term of the sum does not involve a lattice sum is that the corresponding term is $R_{\bar{T} m 2727^{* *}} G_{27^{\prime *} 27^{\prime}} W_{27^{\prime}} \overline{27} s$ with the untwisted intermediat state $27^{\prime}$. For the third term the corresponding term is $R_{\bar{T} m s s^{\prime *}} G_{s^{\prime *} s^{\prime}} W_{27} \overline{27} s^{\prime}$ so the intermediat state $s^{\prime}$ comes from the twisted sector which gives the lattice sum.

As a final result we have

$$
\begin{gathered}
\frac{\partial}{\partial \bar{T}} G(T, \bar{T})=-\frac{\sqrt{2} \pi i}{(T-\bar{T})^{2}}-\frac{\pi}{(T-\bar{T})^{2}} \frac{b^{3} \sqrt{2}}{12 \sqrt{3}} \\
\times \sum_{v \in \Lambda, p \in \Lambda^{*}} e^{2 \pi i\left(\epsilon_{2}-\gamma \alpha v-\gamma \alpha \epsilon_{1}\right) p} w^{Q_{L}} \bar{w}^{Q_{R}} P\left(Q_{R}\right)
\end{gathered}
$$

Solving this differetial equation one can in principle find a solution up to a holomorphic functions of $T$. These functions should transforms under the modular group according (32) and (??). This transformation law implies that the additional terms are modular forms of weight $k=2$ of the subgroup $\Gamma(2)$ of the full modular group. According to [10] they are only of the form $\alpha S+\beta V$, where $S=\theta_{3}^{4}+\theta_{4}^{4}, V=\theta_{2}^{4}$ and $\theta$ 's are theta functions. Using the transformation properties

$$
\begin{aligned}
& \mathcal{T}: S \rightarrow S, V \rightarrow-V \\
& \mathcal{S}: S \rightarrow-\frac{1}{2}(S+3 V), V \rightarrow-\frac{1}{2}(S-V)
\end{aligned}
$$

and requiring the holomorphic ambiguities to satisfy the trasformations (32) and (34) we found that they are of the form

$$
\alpha\left(\begin{array}{c}
S \\
-3 V \\
-3 V \\
-3 S
\end{array}\right)
$$


where $\alpha$ is a some constant. In principle one can find also this constant by taking, for example, limit $T \rightarrow \infty$ of the (28).

\section{Conclusions}

In the present work we give an example of non-zero correlation functions of the type $<27, \overline{27}, 1_{m}, 1_{s}>$, which are related to the additional contribution to the induced mass term for Higgs particles at low energies. We compute such correlation functions explicitly and show that they form finite dimensional representations of the duality group $S L_{2}(Z)$, acting on the untwisted moduli space. The action of the duality group is shown to be consistent with its action on the twisted fields characterized by the fixed points set under the orbifold group. We have also derived the correlation function of bosonic twist fields for the case of $g, g^{-1}, h, h^{-1}$ twisted sectors. Finally, we integrated the correlation functions over 2-dimensional world sheet and discuss ambiguity of the solution using its transformation properties under the action of modular group.

\section{Appendix}

In the presence of the twist fields $\sigma_{g}\left(z_{1}\right) \sigma_{g^{-1}}\left(z_{2}\right) \sigma_{h}\left(z_{3}\right) \sigma_{h^{-1}}\left(z_{4}\right)$ we will need integrals of holomorpic and antiholomorpic functions of the kind I:

$$
\omega(z)=\left(z-z_{1}\right)^{a}\left(z-z_{2}\right)^{c}\left(z-z_{3}\right)^{b}\left(z-z_{4}\right)^{d}
$$

where $a+c=b+d=-1$.

Let's denote $g=e^{2 \pi i a}, h=e^{2 \pi i b}$.

\section{A Integral over complex plane}

We need to evaluate integral $\int_{C} \omega \wedge \omega^{\prime}$ for any two closed one-forms $\omega$ and $\omega^{\prime}$, such that the wedge product of which is well- defined on complex plane (i.e. $\left.g^{\prime}=g^{-1}, h^{\prime}=h^{-1}\right)$.

By Hodge's theorem there exists at least locally a zero-form (function), such that $\omega=d f$. Using Stoke's theorem we have

$$
\int_{C} \omega \wedge \omega^{\prime}=\int_{C} d f \wedge \omega^{\prime}=\int_{C} d\left(f \omega^{\prime}\right)=\int_{\partial C} f \omega^{\prime}
$$

The boundary is shown in Fig. 3. Choosing two independent loops $\mathcal{C}_{1}$ and $\mathcal{C}_{2}$,

\footnotetext{
${ }^{7}$ for the case $g=\theta^{2}$ and $h=\theta^{3}\left(\theta=e^{2 \pi i / 6}\right) a=-2 / 3$ and $b=-1 / 2$
} 


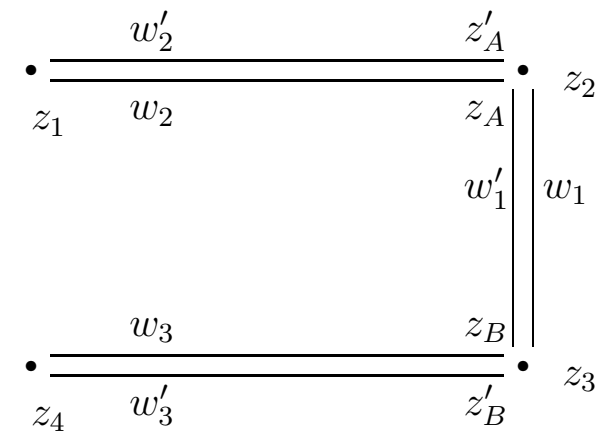

Figure 3: The boundary of the complex plane in the presence of twist fields such that

$$
\begin{gathered}
\oint_{\mathcal{C}_{1}} \omega=\left(g^{-1}-1\right) \int_{z_{1}}^{z_{2}} \omega \\
\oint_{\mathcal{C}_{1}^{-1}} \omega=\left(h^{-1}-1\right) \int_{z_{3}}^{z_{4}} \omega \\
\oint_{\mathcal{C}_{1}} \omega=-\oint_{\mathcal{C}_{1}^{-1}} \omega \\
\oint_{\mathcal{C}_{2}} \omega=(1-g)(1-h) \int_{z_{2}}^{z_{3}} \omega=\gamma \int_{z_{2}}^{z_{3}} \omega
\end{gathered}
$$

we have

$$
\int_{C} \omega \wedge \omega^{\prime}=\left[\oint_{\mathcal{C}_{1}}+\int_{b}+\oint_{\mathcal{C}_{1}^{-1}}+\int_{b^{-1}}\right] f \omega^{\prime}
$$

Using the fact that quantities

$$
\begin{gathered}
f\left(w_{1}\right)-f\left(w_{1}^{\prime}\right)=\left[\int_{w_{1}^{\prime}}^{z_{2}}+\oint_{\mathcal{C}_{1}}+\int_{z_{2}}^{w_{1}}\right] \omega=\oint_{\mathcal{C}_{1}} \omega \\
g f\left(w_{2}^{\prime}\right)-f\left(w_{2}\right)=g\left[f\left(w_{2}\right)+\int_{w_{2}}^{z_{1}} \omega\left(1-g^{-1}\right)\right]-f\left(w_{2}\right)=(g-1) f\left(z_{1}\right) \\
h f\left(w_{3}^{\prime}\right)-f\left(w_{3}\right)=h\left[f\left(w_{3}\right)+\int_{w_{3}}^{z_{3}} \omega\left(1-h^{-1}\right)\right]-f\left(w_{3}\right)=(h-1) f\left(z_{3}\right)
\end{gathered}
$$

doesn't depend on $w$ and

$$
\begin{aligned}
& f\left(z_{A}^{\prime}\right)=f\left(z_{A}\right)+\oint_{\mathcal{C}_{1}} \omega \\
& f\left(z_{B}\right)=f\left(z_{B}^{\prime}\right)+\oint_{\mathcal{C}_{1}^{-1}} \omega \\
& f\left(z_{A}\right)=f\left(z_{B}\right)+\int_{z_{3}}^{z_{2}} \omega
\end{aligned}
$$


we find

$$
\begin{gathered}
{\left[\int_{b}+\int_{b^{-1}}\right] f \omega^{\prime}=\int_{z_{2}}^{z_{3}} \omega^{\prime}\left(w_{1}\right)\left[f\left(w_{1}\right)-f\left(w_{1}^{\prime}\right)\right]=\int_{z_{2}}^{z_{3}} \omega^{\prime} \oint_{\mathcal{C}_{1}} \omega=\frac{1}{\gamma^{\prime}} \oint_{\mathcal{C}_{1}} \omega \oint_{\mathcal{C}_{2}} \omega^{\prime}} \\
{\left[\oint_{\mathcal{C}_{1}}+\oint_{\mathcal{C}_{1}^{-1}}\right] f \omega^{\prime}=\int_{z_{1}}^{z_{2}} \omega^{\prime}\left(w_{2}\right)\left[g f\left(w_{2}^{\prime}\right)-f\left(w_{2}\right)\right]-\int_{z_{3}}^{z_{4}} \omega^{\prime}\left(w_{3}\right)\left[h f\left(w_{3}^{\prime}\right)-f\left(w_{3}\right)\right]} \\
=\frac{g^{\prime}}{1-g^{\prime}}\left[g f\left(z_{A}^{\prime}\right)-f\left(z_{A}\right)\right] \oint_{\mathcal{C}_{1}} \omega^{\prime}+=\frac{h^{\prime}}{1-h^{\prime}}\left[h f\left(z_{B}^{\prime}\right)-f\left(z_{B}\right)\right] \oint_{\mathcal{C}_{1}^{-1}} \omega^{\prime} \\
=\left[f\left(z_{A}\right)-f\left(z_{B}\right)+\oint_{\mathcal{C}_{1}} \omega\left(\frac{1}{1-g^{\prime}} \frac{1}{1-h^{\prime}}\right)\right] \oint_{\mathcal{C}_{1}} \omega^{\prime} \\
=\left[-\frac{1}{\gamma} \oint_{\mathcal{C}_{2}} \omega+\oint_{\mathcal{C}_{1}} \omega\left(\frac{1}{1-h} \frac{1}{1-g}\right)\right] \oint_{\mathcal{C}_{1}} \omega^{\prime}
\end{gathered}
$$

Substituting (42) and (43) into (38) we find

$$
\int_{C} \omega \wedge \omega^{\prime}=\frac{1}{\gamma^{\prime}} \oint_{\mathcal{C}_{1}} \omega \oint_{\mathcal{C}_{2}} \omega^{\prime}-\frac{1}{\gamma} \oint_{\mathcal{C}_{1}} \omega^{\prime} \oint_{\mathcal{C}_{2}} \omega+\left(\alpha-\alpha^{\prime}\right) \oint_{\mathcal{C}_{1}} \omega \oint_{\mathcal{C}_{1}} \omega^{\prime}
$$

where

$$
\alpha=\frac{1}{2}\left(\frac{1}{1-h}-\frac{1}{1-g}\right)
$$

Normalizing $\omega$ and $\omega^{\prime}$ such that

$$
\oint_{\mathcal{C}_{1}} \omega=1, \oint_{\mathcal{C}_{1}} \omega^{\prime}=1
$$

and denoting

$$
\tau=\int_{z_{2}}^{z_{3}} \omega-\alpha, \tau^{\prime}=\int_{z_{2}}^{z_{3}} \omega^{\prime}-\alpha^{\prime}
$$

we get

$$
\int_{C} \omega \wedge \omega^{\prime}=\tau^{\prime}-\tau
$$

Consequently, for

$$
\begin{gathered}
\omega_{1}=z^{a}(z-x)^{c}(z-1)^{b} \\
\omega_{2}=z^{c}(z-x)^{a}(z-1)^{-1-b}
\end{gathered}
$$

where $a+c=-1$, we have

$$
\begin{aligned}
& \int_{C} \omega_{1} \wedge \bar{\omega}_{1}=\bar{\tau}-\tau \\
& \int_{C} \omega_{2} \wedge \bar{\omega}_{2}=\bar{\tau}-\tau
\end{aligned}
$$

since

$$
0=\int_{C} \omega_{1} \wedge \omega_{2}=\tau_{2}-\tau_{1}
$$




\section{B Contour integrals}

If we denote

$$
\omega(a, c, b)=z^{a}(z-x)^{c}(z-1)^{b}
$$

then

$$
\begin{gathered}
\oint_{\mathcal{C}_{1}} \omega(a, c, b)=\left(g^{-1}-1\right) \int_{0}^{x} \omega(a, c, b)=\left(g^{-1}-1\right) e^{-i \pi(b+c)} I_{1}(a, c, b) \\
\oint_{\mathcal{C}_{2}} \omega(a, c, b)=\gamma \int_{x}^{1} \omega(a, c, b)=\gamma e^{-i \pi b} I_{2}(a, c, b)
\end{gathered}
$$

where

$$
\begin{gathered}
I_{1}(a, c, b) \equiv \int_{0}^{x} z^{a}(x-z)^{c}(1-z)^{b} \\
=x^{1+a+c} \frac{\Gamma(a+1) \Gamma(c+1)}{\Gamma(a+c+2)} F(a+1,-b ; a+c+2 ; x) \\
I_{2}(a, c, b) \equiv \int_{x}^{1} z^{a}(z-x)^{c}(1-z)^{b} \\
=(1-x)^{1+c+b} \frac{\Gamma(b+1) \Gamma(c+1)}{\Gamma(c+b+2)} F(b+1,-a ; c+b+2 ; 1-x)
\end{gathered}
$$

(Here $F$ is hypergeometric function.)

Using $g=e^{2 \pi i a}, h=e^{2 \pi i b}, \gamma=(1-g)(1-h)$ we find

$$
\begin{gathered}
\oint_{\mathcal{C}_{1}} \omega(a, c, b)=-2 i e^{-i \pi(a+c+b)} s(a) x^{1+a+c} \\
\times \frac{\Gamma(a+1) \Gamma(c+1)}{\Gamma(a+c+2)} F(a+1,-b ; a+c+2 ; x) \\
\oint_{\mathcal{C}_{2}} \omega(a, c, b)=-4 e^{i \pi a} s(a) s(b)(1-x)^{1+c+b} \\
\times \frac{\Gamma(b+1) \Gamma(c+1)}{\Gamma(c+b+2)} F(b+1,-a ; c+b+2 ; 1-x)
\end{gathered}
$$

where $s(a)=\sin (\pi a)$. In the case $a+c=-1$ (46) becomes

$$
\begin{gathered}
\oint_{\mathcal{C}_{1}} \omega(a,-1-a, b)=-2 i e^{-i \pi b} \pi F(a+1,-b ; 1 ; x) \\
\oint_{\mathcal{C}_{2}} \omega(a,-1-a, b)=-4 e^{i \pi a} s(a) s(b)(1-x)^{b-a}
\end{gathered}
$$




$$
\times \frac{\Gamma(b+1) \Gamma(-a)}{\Gamma(b-a+1)} F(b+1,-a ; b-a+1 ; 1-x)
$$

\section{Acknowledgements}

I would like to express the gratitude and my special thanks to Prof. K.Narain for his help and useful discussions.

This work was supported by the International Centre for Theoretical Physics, Trieste, the International Atomic Energy Agency, and the United Nations Educational, Scientific and Cultural Organization.

\section{References}

[1] L.J.Dixon, V.S.Kalunovsky and J.Louis, Nucl. Phys. B329 (1990) 27

[2] I.Antoniadis, E.Gava, K.S.Narain and T.R.Taylor, preprint, ICTP $\mathrm{IC} / 94 / 72$

[3] G.F.Giudice and A.Masiero, Phys. Lett. B206 (1988) 480

[4] D.J.Gross, J.A.Harvey, E.Martinec and R.Rohm, Nucl. Phys. B256 (1985) 253

[5] L.Dixon, D.Friedan, E.Martinec and S.Shenker, Nucl. Phys. B282 (1987) 13

[6] L.Dixon, J.Harvay, C.Vafa and E.Witten, Nucl. Phys. B261 (1985) 678

[7] D.Friedan, E.Martinec and S.H.Shenker, Nucl. Phys. B271 (1986) 93

[8] K.S.Narain, M.H.Sarmadi and C.Vafa, Nucl. Phys. B356 (1991) 163

[9] I.Antoniadis, K.S.Narain and T.R.Taylor, Phys. Lett. B267 (1991) 37

[10] R.A.Rankin, Modular forms and functions, Cambridge University Press, 1977

[11] I.S.Gradshtein and I.M.Ryznik, Table of integrals, series and products, Academic Press, 1980 
This figure "fig1-1.png" is available in "png" format from: http://arxiv.org/ps/hep-th/9502046v1 